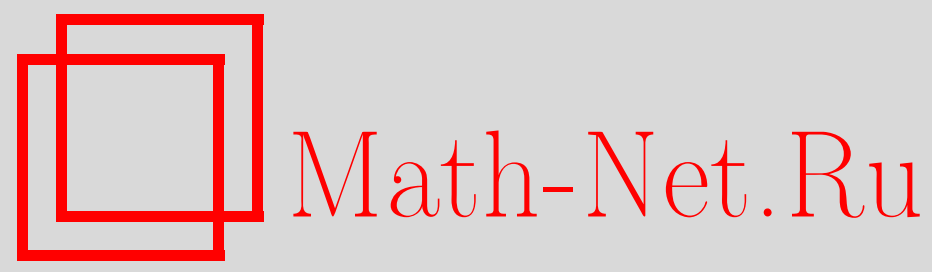

А. М. Воронцов, О совместных приближениях в банаховых пространствах обобщенных функций, Матем. заметкu, 2003, том 73, выпуск 2, 179-194

DOI: https://doi.org/10.4213/mzm188

Использование Общероссийского математического портала Math-Net.Ru подразумевает, что вы прочитали и согласны с пользовательским соглашением http://www . mathnet.ru/rus/agreement

Параметры загрузки:

IP: 54.166 .219 .16

26 апреля 2023 г., 12:52:11

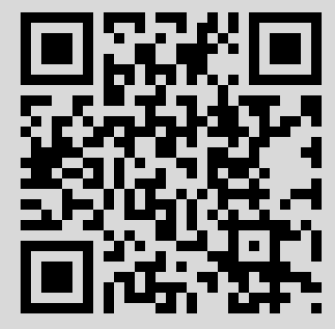




\title{
О СОВМЕСТНЫХ ПРИБЛИЖЕНИЯХ В БАНАХОВЫХ ПРОСТРАНСТВАХ ОБОБЩЕННЫХ ФУНКЦИЙ
}

\author{
А. М. Воронцов
}

\begin{abstract}
Для заданного однородного эллиптического оператора $L$ в частных производных с постоянными комплексными коэффициентами, двух банаховых пространств $V_{1}$ и $V_{2}$ обобщенных функций в $\mathbb{R}^{N}$ и компактных множеств $X_{1}$ и $X_{2}$ в $\mathbb{R}^{N}$ исследуются совместные аппроксимации в нормах пространств $V_{1}\left(X_{1}\right)$ и $V_{2}\left(X_{2}\right)$ (пространств струйраспределений по Уитни) посредством решений уравнения $L u=0$ в окрестностях множества $X_{1} \cup X_{2}$. Получена локализационная теорема, позволяющая в определенных условиях сводить указанную аппроксимационную задачу к соответствующим задачам в каждом из пространств по отдельности.

Библиограбой: 17 названий.
\end{abstract}

\section{1. Введение}

В теории приближений функций решениями однородных эллиптических уравнений $L u=0$ на компактах $X$ в $\mathbb{R}^{N}$ аппроксимации исследуются как в классических топологиях (например, в нормах пространств $C^{m}(X), \operatorname{Lip}_{\alpha}(X), L_{p}(X)$ или в топологии ограниченно-поточечной сходимости), так и в “абстрактных", т.е. в топологиях произвольных подпространств пространства обобщенных функций $\left(C_{0}^{\infty}\left(\mathbb{R}^{N}\right)\right)^{*}$ с определенным набором условий. При этом, как правило, аппроксимации ведутся в одной фиксированной норме, обладающей какими-либо свойствами однородности и инвариантности. Такие приближения не могут учитьвать различные свойства гладкости приближаемых функций на разных подмножествах в $X$. Таким образом, весьма естественно возникает задача о совместной (одновременной) апроксимации в нескольких, для начала в двух, различных нормах. Этой тематике посвящен ряд работ (см., например, [1]-[3] и цитируемые там работы), где соответствуюшие нормы или топологии являются классическими: равномерная, поточечно-ограниченная, липшицева. В контексте абстрактных пространств аппроксимации, насколько это известно автору, данная задача рассматривается впервые. В принципе, возможен целый ряд различных постановок этой задачи. Наиболее естественным на наш взгляд является "уитниевский” подход, развитый в контексте абстрактных аппроксимаций в одной норме в работах [4], [5]. Основной целью

Работа выполнена при поддержке Российского фонда фундаментальных исследований, грант № 00-01-00618. 
настоящей работы является получение “абстрактной” локализационной теоремы (теоремы 3), сводящей задачу совместной аппроксимации в двух нормах к задачам апшроксимации в каждой из этих норм по отдельности (эти задачи считаются априори решенными). Главная трудность здесь состоит в том, что соответствующий локализационный оператор Витушкина, играющий существенную роль в таких задачах, как правило, оказывается неинвариантньм в естественным образом возникающих пространствах аппроксимации. Локализационная теорема получена пока при некоторых ограничениях на множество $X=X_{1} \cup X_{2}$. Аналогичные ограничения возникают уже в простейших случаях равномерной рациональной апшроксимации (см. [6]), когда $V_{1}=V_{2}=C\left(\mathbb{R}^{2}\right)$, а приближения исследуются на объединении двух компактов, на каждом из которых нужная апшроксимация имеет место.

Приведем для наглядности два следствия из упомянутой локализационной теоремы (ее полная формулировка достаточно громоздка и требует введения целого ряда обозначений). Фиксируем произвольные $\alpha$ и $\beta$, удовлетворяюшие условию $0<\alpha<\beta<1$. Рассмотрим задачу о совместных приближениях в пространствах $V_{1}=\operatorname{lip}_{\alpha}$ и $V_{2}=\operatorname{lip}_{\beta}$ (подробнее см. п. 4.1), причем в качестве эллиптического оператора $L$ берется оператор Коши-Римана $L=\partial / \partial \bar{z}$ в $\mathbb{C}_{z}$. Пусть $X_{1}$ и $X_{2}$ - компакты в $\mathbb{C}, X=X_{1} \cup X_{2}$, $X_{0}=X_{1} \cap X_{2} \neq \varnothing$. Положим

$$
V_{1,2}=\left\{\left(f_{1}, f_{2}\right): f_{1} \in \operatorname{lip}_{\alpha}\left(X_{1}\right), f_{2} \in \operatorname{lip}_{\beta}\left(X_{2}\right),\left.f_{1}\right|_{X_{0}}=\left.f_{2}\right|_{X_{0}}\right\}
$$

с нормой

$$
\left\|\left(f_{1}, f_{2}\right)\right\|_{1,2}=\max \left\{\left\|f_{1}\right\|_{V_{1}\left(X_{1}\right)},\left\|f_{2}\right\|_{V_{2}\left(X_{2}\right)}\right\},
$$

и пусть еше $H_{1,2}$ есть подпространство в $V_{1,2}$, состоящее из тех $\left(f_{1}, f_{2}\right)$, для которых $f_{s}$ голоморфнана $\stackrel{\circ}{X}_{s}, s \in\{1,2\}$.

Tеорема 1. Пусть $X_{1}$ и $X_{2}$ - компакты в $\mathbb{C}$ с условием $\overline{\left(X_{1} \backslash X_{2}\right)^{\circ}} \cap X_{2}=\varnothing$. Следующие условия әквивалентны:

(i) для всякой пары $\left(f_{1}, f_{2}\right)$ из $H_{1,2}$ и любого $\varepsilon>0$ найдется функиия $f$, голоморфная в некоторой окрестности $X$, для которой выполнено условие $\left\|\left(f_{1}-f, f_{2}-f\right)\right\|_{1,2}<\varepsilon$

(ii) найдутся $p \geqslant 1$ u $A>0$ такие, что для всякого открытого круга $B(z, \delta) c$ условием $\bar{B}(z, \delta) \cap X_{2}=\varnothing$ выполняется

$$
M_{*}^{1+\alpha}\left(B(z, \delta) \backslash \stackrel{\circ}{X}_{1}\right) \leqslant A M^{1+\alpha}\left(B(z, p \delta) \backslash X_{1}\right),
$$

и для всякого открытого круга $B(z, \delta)$ имеет место

$$
M_{*}^{1+\beta}\left(B(z, \delta) \backslash \stackrel{\circ}{X}_{2}\right) \leqslant A M^{1+\beta}\left(B(z, p \delta) \backslash X_{2}\right) ;
$$

(iii) условия предыдущего пункта выполняются для $p=1$.

Здесь $M^{1+\alpha}(\cdot)\left(\right.$ соответственно $\left.M_{*}^{1+\alpha}(\cdot)\right)$ - обхват (соответственно нижний обхват) по Хаусдорфу порядка $1+\alpha$ (см. [7]).

Приведем еще одно следствие локализационной теоремы для совместных равномерных и $C^{1}$-приближений. Для произвольного компакта $K$ в $\mathbb{C}$ через $C^{1}(K)$ обозначим совокупность троек $f=\left(f_{0}, f_{1}, f_{2}\right)$ таких, что для $f$ найдется $F \in C^{1}(\mathbb{C})$, для которой выполнены условия

$$
\left.F\right|_{K}=f_{0},\left.\quad F_{x}^{\prime}\right|_{K}=f_{1},\left.\quad F_{y}^{\prime}\right|_{K}=f_{2}, \quad z=x+i y .
$$

Уитниевская норма $\|f\|_{1, K}$ в $C^{1}(K)$ задается как нижняя грань норм $\|F\|_{1}$ в $C^{1}(\mathbb{C})$ (норма стандартная, см. п. 4.1 пример 2) среди всех $F$ с указанным выше свойством. 
Tеорема 2. Пусть $X_{1}$ и $X_{2}-$ компакты в $\mathbb{C}$ с условием $\overline{\left(X_{1} \backslash X_{2}\right)^{\circ}} \cap X_{2}=\varnothing$. Следующие условия эквивалентны:

(i) для любих $f=\left(f_{0}, f_{1}, f_{2}\right) \in C^{1}\left(X_{2}\right)$ u $g \in C\left(X_{1}\right)$ таких, что $f_{0}=g$ на $X_{1} \cap X_{2}, g$ голоморфнана $\dot{X}_{1}, f_{0}$ голоморфна на $\dot{X}_{2}$, и любого $\varepsilon>0$ найдетcя $h$, голоморфная в некоторой окрестности $X_{1} \cup X_{2}$, для которой

$$
\|g-h\|_{C\left(X_{1}\right)}<\varepsilon \quad u \quad\left\|f-\left(h, h_{x}^{\prime}, h_{y}^{\prime}\right)\right\|_{1, X_{2}}<\varepsilon
$$

(ii) $\stackrel{\circ}{X}_{2}$ плотно в $X_{2}$ и, кроме того, найдутся $p \geqslant 1 u A>0$ такие, что для каждого открытого круга $B(z, \delta)$ с условием $\bar{B}(z, \delta) \cap X_{2}=\varnothing$ имеет место оценка

$$
\alpha\left(B(z, \delta) \backslash \stackrel{\circ}{X}_{1}\right) \leqslant A \alpha\left(B(z, p \delta) \backslash X_{1}\right) ;
$$

(iii) $\stackrel{\circ}{X}_{2}$ плотно в $X_{2}$ u, кроме того, для каждого открытого круга $B(z, \delta) c$ условием $\bar{B}(z, \delta) \cap X_{2}=\varnothing$ имеет место равенство

$$
\alpha\left(B(z, \delta) \backslash \stackrel{\circ}{X}_{1}\right)=\alpha\left(B(z, \delta) \backslash X_{1}\right) .
$$

Здесь $\alpha(\cdot)$ - аналитическая $C$-емкость (см. [8]).

В разделе 2 даются необходимые предварительные сведения и формулируется задача о совместной аппроксимации для двух норм, одна из которьх "сильнее" другой.

В разделе 3 приводится формулировка основного результата (теоремы 3 и 4 ) и его доказательство. Здесь существенно используются методы, развитые в работах [9], [4], [5].

В разделе 4 приводятся конкретные примеры использования теоремы 3 , в частности, доказываются теоремы 1 и 2 из раздела 1 . Для случая аппроксимации на нигде не плотных компактах весьма полезным является предложение 6 , представляющее собой обобщение (абстрактный вариант) аналогичных результатов из [10]-[12].

\section{2. Предварительные сведения}

2.1. Банаховы пространства $V, V(X), V_{\text {lос }}$ Пусть $V$-комплексное банахово пространство с нормой $\|\cdot\|$, которое содержит $C_{0}^{\infty}\left(\mathbb{R}^{N}\right)=C_{0}^{\infty}$ - пространство основных (комплекснозначных) функций и само содержится в $\left(C_{0}^{\infty}\right)^{*}$ - пространстве обобщенных функций (распределений). Предположим также, что $V$ наделено структурой топологического $C_{0}^{\infty}$-подмодуля в $\left(C_{0}^{\infty}\right)^{*}$. Последнее означает, что при всех $f \in V$ и $\varphi \in C_{0}^{\infty}$ имеет место $\varphi f \in V$, причем

$$
\begin{array}{r}
\|\varphi f\| \leqslant A(\varphi)\|f\|, \\
|\langle f, \varphi\rangle| \leqslant A(\varphi)\|f\|,
\end{array}
$$

где $\langle f, \varphi\rangle$ - действие распределения $f$ на основную функцию $\varphi$, а $A(\varphi)$ не зависит от $f$.

Можно показать [5], что из указанных свойств вытекает непрерьвность вложений $C_{0}^{\infty} \hookrightarrow V$ и $V \hookrightarrow\left(C_{0}^{\infty}\right)^{*}$. Придерживаясь терминологии, предложенной О'Фарреллом [9], будем назьвать такие пространства $V$ конкретными банаховыми пространствами (КБП).

Для компактного множества $X$ в $\mathbb{R}^{N}$ обозначим через $I(X)$ замыкание в $V$ линейного подмногообразия $\{f \in V: \operatorname{supp} f \cap X=\varnothing\}$, где $\operatorname{supp} f$ - носитель распределения $f$. 
Банахово пространство $V(X):=V / I(X)$, наделенное факторнормой, рассматривается как наиболее естественное (типа Уитни) “сужение" $V$ на $X$.

Пусть $\|f\|_{X}=\inf \left\{\left\|f+f_{0}\right\|: f_{0} \in I(X)\right\}$ - норма элемента ("струи") $f_{(X)}=f+I(X)$ в $V(X)$, определенного распределением $f \in V$.

Для произвольного открытого множества $D$ в $\mathbb{R}^{N}$ определим

$$
V_{\mathrm{loc}}(D)=\left\{f \in\left(C_{0}^{\infty}(D)\right)^{*}: f \varphi \in V \text { для всех } \varphi \in C_{0}^{\infty}(D)\right\}
$$

где полагается $f \varphi=0$ вне $D$ (при этом $f$ - обобщенная функция в $D$ ). Через $V_{\text {lос будем }}$ обозначать $V_{\text {loc }}\left(\mathbb{R}^{N}\right)$. В пространстве $V_{\text {loc }}(D)$ известным образом вводится топология Фреше (см. [4, с. 253]).

Если $X$-компакт в $D$ и $f \in V_{\text {loc }}(D)$, то корректно определен элемент $f_{(X)}$. Аналогично, для любого компакта $X^{\prime} \subseteq X$ и $f_{(X)} \in V(X)$ естественным образом определяется $\|f\|_{X^{\prime}}$, причем $\|f\|_{X^{\prime}} \leqslant\|f\|_{X}$ (подробнее о КБП см. [9] и [5]).

2.2. Эллиптический оператор $L$. Пусть $\alpha=\left(\alpha_{1}, \ldots, \alpha_{N}\right)$ - мультииндекс $\alpha_{j} \in$ $\mathbb{Z}_{+}=\{0,1,2, \ldots\}$. Положим $|\alpha|=\alpha_{1}+\cdots+\alpha_{N}, \alpha !=\alpha_{1} ! \cdots \alpha_{N} !, x^{\alpha}=x_{1}^{\alpha_{1}} \cdots x_{N}^{\alpha_{N}}$ при $x=\left(x_{1}, \ldots, x_{N}\right) \in \mathbb{R}^{N}$ и

$$
\partial^{\alpha}=\frac{\partial^{|\alpha|}}{\partial x_{1}^{\alpha_{1}} \cdots \partial x_{N}^{\alpha_{N}}} .
$$

Всюду далее $L(\xi)=\sum_{|\alpha|=r} a_{\alpha} \xi^{\alpha}-$ некоторьй полином степени $r \geqslant 1$ с постоянными комплексными коэффициентами $a_{\alpha}$, удовлетворяющий условию эллиптичности: $L(\xi) \neq 0$ при всех $\xi \neq 0, \xi \in \mathbb{R}^{N}$. Ассоциируем с $L(\xi)$ однородньй эллиптический оператор

$$
L=L(\partial)=\sum_{|\alpha|=r} a_{\alpha} \partial^{\alpha}
$$

порядка $r$. Хорошо известно, что для такого оператора $L$ существует специальное фундаментальное решение $\Phi$, приведенное, например, в [13, теорема 7.1.20]. Обозначим через $B(a, \delta)$ (соответственно $\bar{B}(a, \delta))$ открытьй (соответственно замкнутьй) шар с центром $a \in \mathbb{R}^{N}$ и радиусом $\delta>0$. Если $B=B(a, \delta)$ и $\theta>0$, то положим $\theta B=B(a, \theta \delta)$ и $\theta \bar{B}=\bar{B}(a, \theta \delta)$. Пусть $\varphi \in C_{0}^{\infty}$. Локализачионный оператор Витушкина $\mathscr{V}_{\varphi}:\left(C_{0}^{\infty}\right)^{*} \rightarrow$ $\left(C_{0}^{\infty}\right)^{*}$, ассоииированны й с $L$ и $\varphi$, определяется по формуле $\mathscr{V}_{\varphi} f=\Phi *(\varphi L f)$, где $*$ - оператор свертки. Скажем, что $\mathscr{V}_{\varphi}$ действует инвариантно на $V_{\mathrm{loc}}$, если $\mathscr{V}_{\varphi}: V_{\mathrm{loc}} \rightarrow V_{\mathrm{loc}}$ и это отображение непрерьвно. Последнее эквивалентно тому, что для всякого открытого шара $B$, содержащего $\operatorname{supp} \varphi$, и для всех $f \in V_{\text {loc }}$ имеет место $\mathscr{V}_{\varphi} f \in V_{\text {loc }}$ и

$$
\left\|\mathscr{V}_{\varphi} f\right\|_{\bar{B}} \leqslant A(\varphi, B)\|f\|_{\bar{B}}
$$

где $A(\varphi, B)$ не зависит от $f$.

Надо отметить, что последнее условие вьполняется для любого эллиптического оператора $L$ и всякого $V$, локально эквивалентного какому-либо "классическому" пространству функций в $\mathbb{R}^{N}[9]$. 
2.3. $L$-аналитические функции и ряды Лорана. Пусть $D$ - открытое множество в $\mathbb{R}^{N}$. Через $L(D)$ обозначается класс распределений $f$ в $D$, для которых $L f=0$ в $D$ в смысле теории распределений. Из леммы Вейля [14, следствие 11.4.13] следует, что $L(D) \hookrightarrow C^{\infty}(D)$, т.е. всякое обобшенное решение является классическим, откуда $L(D) \subset V_{\text {loc }}(D)$. Далее, если $\left\{f_{m}\right\}$ - последовательность в $L(D)$ такая, что $f_{m} \rightarrow f$ в $V_{\text {loc }}(D)$ при $m \rightarrow \infty$, то $f \in L(D)$, поскольку сходимость в $V_{\text {loc }}(D)$ сильнее сходимости в $\left(C_{0}^{\infty}\right) *$, а для нее последнее утверждение верно. Следуя [5], назовем функции из $L(D)$ $L$-аналитическими в $D$.

Для компактного множества $X$ в $\mathbb{R}^{N}$ обозначим через $L(X)$ класс функций $f$, каждая из которых определена и удовлетворяет уравнению $L f=0$ в некоторой (для каждой $f$ своей) окрестности $X$, а через $L_{V}(X)$ замыкание в $V(X)$ линейного подмногообразия $L(X)_{(X)}=\left\{f_{(X)}: f \in L(X)\right\}$. Таким образом, $L_{V}(X)$ есть класс элементов (струй) из $V(X)$, допускающих приближение в норме $\|\cdot\|_{X}$ струями, определенньми $L$-аналитическими в окрестности $X$ функциями.

Справедлив следующий фундаментальньй факт (см., например, [7, с. 163]): найдется $k>1$ такое, что если $T$ - распределение с компактным носителем в $B(a, \delta)$ и $f=\Phi * T$, то при $|x-a|>k \delta$ справедливо следующее разложение лорановского типа:

$$
f(x)=\langle T(y), \Phi(x-y)\rangle=\sum_{|\alpha| \geqslant 0} c_{\alpha} \partial^{\alpha} \Phi(x-a)
$$

где

$$
c_{\alpha}=(-1)^{\alpha}(\alpha !)^{-1}\left\langle T(y),(y-a)^{\alpha}\right\rangle
$$

причем указанный ряд сходится в смысле $C^{\infty}(|x-a|>k \delta)$.

\section{3. Теоремы о совместных приближениях}

3.1. Постановка задачи о совместных приближениях. Пусть $V_{1}$ и $V_{2}-$ КБП с нормами $\|\cdot\|_{1}$ и $\|\cdot\|_{2}$ соответственно. Для произвольного компакта $X$ в $\mathbb{R}^{N}$ и $s \in\{1,2\}$ обозначим через $I_{s}(X)$ замыкание в $V_{s}$ линейного подмногообразия $\left\{f \in V_{s}: \operatorname{supp} f \cap\right.$ $X=\varnothing\}$, а при $f_{s} \in V_{s}$ определим $f_{s(X)}^{(s)}:=f_{s}+I_{s}(X)$ - элемент (струя) из $V_{s}(X)$,

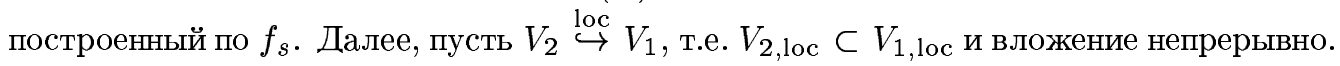
Последнее означает, что для любого компакта $X$ найдется компакт $Y \supset X$ такой, что

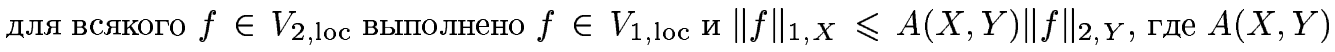
не зависит от $f$. Отметим, что условие локального вложения $V_{2} \stackrel{\text { loc }}{\hookrightarrow} V_{1}$ позволяет для произвольного компакта $X$ корректно определить по элементу $F_{2} \in V_{2}(X)$ элемент $F_{1} \in V_{1}(X)$ с условием $F_{1}=F_{2}^{(1)}$.

Уточним также, что при $F_{s} \in V_{s}(X)$ условие $F_{s} \in L(\stackrel{\circ}{X})$ означает, что $f_{s} \in L(\stackrel{\circ}{X})$ для любого представителя $f_{s}$ струи $F_{s}$. Здесь $\stackrel{X}{X}$ - внутренность $X$.

Теперь фиксируем произвольные непустые компакты $X_{1}$ и $X_{2}$ в $\mathbb{R}^{N}$ и обозначим $X:=$ $X_{1} \cup X_{2}, X_{0}:=X_{1} \cap X_{2}$. Пусть $L$ - эллиптический оператор порядка $r$ (как в п. 2.2). Введем в рассмотрение банахово пространство

$$
V_{1,2}:=V_{1,2}\left(X_{1}, X_{2}\right):=\left\{F=\left(F_{1}, F_{2}\right) \mid F_{s} \in V_{s}\left(X_{s}\right) \text { при } s \in\{1,2\} \text { и } F_{1}^{(1)}\left(X_{0}\right)=F_{2}^{\left(X_{0}\right)}\right\}
$$


с нормой

$$
\|F\|_{1,2}=\max _{s \in\{1,2\}}\left\{\left\|F_{s}\right\|_{s, X_{s}}\right\}
$$

а также его подпространства

$$
\begin{aligned}
& H_{1,2}:=H_{1,2}\left(X_{1}, X_{2}\right):=\left\{\left(F_{1}, F_{2}\right) \mid\left(F_{1}, F_{2}\right) \in V_{1,2} \text { и } F_{s} \in L\left(\dot{X}_{s}\right) \text { при } s \in\{1,2\}\right\}, \\
& W_{1,2}:=W_{1,2}\left(X_{1}, X_{2}\right):=\left\{\left(F_{1}, F_{2}\right) \mid\left(F_{1}, F_{2}\right) \in V_{1,2} \text { и } F_{s} \in L_{V_{s}}\left(X_{s}\right) \text { при } s \in\{1,2\}\right\} .
\end{aligned}
$$

Определим подпространство $L_{1,2}:=L_{1,2}\left(X_{1}, X_{2}\right)$ как замыкание в $V_{1,2}$ линейного подмногообразия $\left\{\left(g_{\left(X_{1}\right)}^{(1)}, g_{\left(X_{2}\right)}^{(2)}\right): g \in L(X)\right\}$. Ясно, что $L_{1,2} \subseteq W_{1,2} \subseteq H_{1,2} \subseteq V_{1,2}$ и $L_{1,2}$ является подпространством пар из $V_{1,2}$, допускающих одновременную (или совместную) аппроксимацию в норме $\|\cdot\|_{1,2}$ струями, $L$-аналитическими в окрестности $X$. Априори мы будем считать, что пространства $L_{V_{s}}\left(X_{s}\right), s \in\{1,2\}$, в достаточной степени изучены, т.е. условия приближаемости $L$-аналитическими функциями на компактах в $\mathbb{R}^{N}$ в каждом из пространств $V_{s}$ по отдельности для нас известны.

Наша основная задача о совместных приближениях $L$-аналитическими функциями на компактах в $\mathbb{R}^{N}$ состоит в следующем.

ЗАдАчА 1 . Найти условия на $F_{s} \in V_{s}\left(X_{s}\right)$ (а также на $\left.X_{s}, V_{s}, L\right), s \in\{1,2\}$, необходимые и достаточные для того, чтобы $\left(F_{1}, F_{2}\right) \in L_{1,2}$.

Естественным необходимым условием приближаемости является $\left(F_{1}, F_{2}\right) \in W_{1,2}$. Однако это условие, вообще говоря, не является достаточным, поэтому возникает следующая задача.

ЗАДАчА 2. Каковы условия на $X_{s}, V_{s}$ и $L, s \in\{1,2\}$, необходимые и достаточные для того, чтобы $W_{1,2}=L_{1,2}$ ?

В данной работе будет найден ряд достаточных условий для выполнения последнего равенства, а также приведены конкретные примеры наличия аппроксимации.

Перейдем к точным формулировкам.

3.2. Формулировка основного результата. Пусть, как и вьше, $X_{1}$ и $X_{2}$-непустые компакты в $\mathbb{R}^{N}$. Будем далее считать, что $X_{0}=X_{1} \cap X_{2} \neq \varnothing$; иначе задачи 1 и 2 бессодержательны. Введем два условия, которые будут важны в дальнейшем.

УСловиЕ $(\mathrm{K})$ ("существование коридора"). Скажем, что пара $\left(X_{1}, X_{2}\right)$ удовлетворяет условию $(\mathrm{K})$, если $\overline{\left(X_{1} \backslash X_{2}\right)^{\circ}} \cap X_{2}=\varnothing$.

Введем обозначение: $Y_{1,2}=\overline{\left(X_{1} \backslash X_{2}\right)^{\circ}}$. Также нам потребуется следующее

УСловиЕ (ПК) ( "приближаемость в коридоре"). Пара $\left(X_{1}, X_{2}\right)$ с условием $(\mathrm{K})$ удовлетворяет условию $(\Pi \mathrm{K})$, если найдется компакт $Y_{0}, Y_{1,2} \subset Y_{0} \subset\left(X_{1} \backslash X_{2}\right)$, такой, что для каждого $x \in\left(X_{1} \backslash X_{2}\right) \backslash Y_{0}$ существуют $\delta_{x}>0$ и $p_{x} \geqslant 1$, для которых $V_{1}\left(\bar{B}\left(x, p_{x} \delta_{x}\right) \cap X_{1}\right) \subset L_{V_{1}}\left(\bar{B}\left(x, \delta_{x}\right) \cap X_{1}\right)$.

Отметим, что условие (ПК) также включает ограничения на $\left(V_{1}, L\right)$. В работе [5] приведены примеры, показьвающие, что даже в классических случаях $p_{x}$ может быть строго больше единицы. 
ЗАМЕЧАнИЕ 1. Из условия (ПК) следует [5, §4, предложение 1$]$, что для любого $x \in\left(X_{1} \backslash X_{2}\right) \backslash Y_{0}$ и для любого $p_{x}>1$ найдется $\delta_{x}>0$ такое, что при любом $\delta \in\left(0, \delta_{x}\right)$ имеем $V_{1}\left(\bar{B}\left(x, p_{x} \delta\right) \cap X_{1}\right) \subset L_{V_{1}}\left(\bar{B}(x, \delta) \cap X_{1}\right)$.

Теперь мы можем сформулировать основной результат этой работы.

ТЕОРемА 3. Пусть $V_{1} u V_{2}-$ КБП, $\left(V_{1}, L\right)$ удовлетворяет оценке $(3) u V_{2} \stackrel{\text { loc }}{\hookrightarrow} V_{1}$. Пусть $X_{1}$ и $X_{2}$ - непустые компакты в $\mathbb{R}^{N}$ с условием (ПК). Тогда $W_{1,2}=L_{1,2}$.

Непосредственным следствием теоремы 3 и [4, теорема 3$]$ является

Теорема 4. При выполнении условий теоремы 3 пусть $F=\left(F_{1}, F_{2}\right) \in V_{1,2}$. Тогда следуюшие условия әквивалентны:

(i) $F \in L_{1,2}$;

(ii) для всех открытых шаров $B$ в $\mathbb{R}^{N}$ имеет место включение

$$
F_{s} \in L_{V_{s}}\left(\bar{B} \cap X_{s}\right), \quad s \in\{1,2\} ;
$$

(iii) найдется (конечное) покрытие $\left\{B_{j}\right\}_{j=1}^{J}$ компакта $X$ открытыми шарами $B_{j}$ такое, что для каждого $j \in\{1, \ldots, J\}$ выполнено одно из двух условий:

(a) $B_{j} \cap Y_{0}=\varnothing, B_{j} \cap X_{2} \neq \varnothing$, причем $F_{2} \in L_{V_{2}}\left(\bar{B}_{j} \cap X_{2}\right)$;

(b) $B_{j} \cap Y_{0} \neq \varnothing, B_{j} \cap X_{2}=\varnothing$, причем $F_{1} \in L_{V_{1}}\left(\bar{B}_{j} \cap X_{1}\right)$.

Предпошлем доказательству теоремы 3 несколько вспомогательных утверждений.

3.3. Вспомогательные утверждения. Пусть всюду далее $V_{1}$ и $V_{2}-\mathrm{KБП,} X_{1}$ и $X_{2}$ - компакты, $X_{0}=X_{1} \cap X_{2} \neq \varnothing$.

ПРЕДЛОЖЕНИЕ 1. Пусть $\left(F_{1}, F_{2}\right) \in V_{1,2}$. Тогда для любого компакта $Y \subset\left(X_{1} \backslash X_{2}\right)$ и числа $\varepsilon>0$ найдутся окрестность $U_{2}$ компакта $X_{2}$ и $f_{0} \in V_{1}$, удовлетворяющие следуюшим условиям:

1) $\operatorname{supp} f_{0}-$ компакт;

2) $f_{0} \in V_{2, \text { loc }}\left(U_{2}\right)$;

3) $f_{0}\left(X_{2}\right)=F_{2}, f_{0}^{(1)}(1)=F_{1(Y)}^{(1)}$

4) $\left\|\left(f_{0}, f_{0}\right)-\left(F_{1}, F_{2}\right)\right\|_{1,2}<\varepsilon$.

ЗАмЕчАниЕ 2. Здесь и далее, под $\left(f_{0}, f_{0}\right)$, для подходящего $f_{0}$, мы понимаем элемент $\left(f_{0}\left(X_{1}\right), f_{0}^{(2)}\left(X_{2}\right)\right)$ из $V_{1,2}$, а под разностью $F=\left(F_{1}, F_{2}\right)$ и $G=\left(G_{1}, G_{2}\right)$ из $V_{1,2}$ понимаем элемент $F-G=\left(F_{1}-G_{1}, F_{2}-G_{2}\right)$, также принадлежащий $V_{1,2}$.

ДОКАЗАТЕЛЬСТво ПРЕДЛОЖЕНИЯ 1. Фиксируем $\varepsilon>0$. Пусть $f_{1} \in F_{1}$ и $f_{2} \in F_{2}$ некоторые представители классов $F_{1}$ и $F_{2}$ соответственно. Из условия $\left(F_{1}, F_{2}\right) \in V_{1,2}$ получаем, что $\left(f_{1}-f_{2}\right) \in I_{1}\left(X_{0}\right)$. Следовательно, для каждого $\delta>0$ найдется распределение $h_{\delta} \in V_{1}$ такое, что $\left\|h_{\delta}\right\|_{1}<\delta$ и $f_{1}-f_{2}+h_{\delta}=0$ в окрестности $X_{0}$. Фиксируем функцию $\varphi \in C_{0}^{\infty}$ такую, что $\varphi \equiv 0$ в некоторой окрестности $Y, \varphi \equiv 1$ в некоторой окрестности $U_{2}$ компакта $X_{2}$. Используя (2) для $V_{1}$, получаем, что $\left\|h_{\delta} \varphi\right\|_{1} \leqslant A(\varphi)\left\|h_{\delta}\right\|_{1}<\varepsilon$ при достаточно малом $\delta>0$. Ясно, что $f_{1}-f_{2}+h_{\delta} \varphi=0$ в окрестности $X_{0}$, и поэтому найдется $h_{0} \in V_{1}$ со свойствами $h_{0}=f_{1}-f_{2}+h_{\delta} \varphi$ в окрестности $X_{1}$ и $h_{0}=0$ в окрестности $X_{2}$. Положим $f_{0}=\left(h_{0}+f_{2}\right) \psi$, где $\psi \in C_{0}^{\infty} ; \psi \equiv 1$ в окрестности $X$. Тогда 
$f_{0} \in V_{1}, f_{0}=f_{2}$ в $U_{2}, f_{0}=f_{1}+h_{\delta} \varphi$ в окрестности $X_{1}$ и, следовательно, $f_{0}=f_{1}$ в окрестности $Y$, причем $\left\|\left(f_{0}, f_{0}\right)-\left(F_{1}, F_{2}\right)\right\|_{1,2}=\left\|h_{\delta} \varphi\right\|_{1, X_{1}}<\varepsilon$. Следовательно, $f_{0}-$ нужное распределение.

Таким образом, предложение 1 показывает, что решение задачи 1 для пары $\left(F_{1}, F_{2}\right) \in$ $V_{1,2}$ вытекает из решения этой же задачи для пары $\left(f_{0}, f_{0}\right)$, построенной указанным способом по $\left(F_{1}, F_{2}\right)$. Следующие предложения 2 и 3 дополняют предложение 1 в случае, когда $F_{s} \in L_{V_{s}}\left(X_{s}\right)$ при $s=1$ или 2.

ПРЕДЛОЖЕнИЕ 2. Пусть $V_{2} \stackrel{\text { loc }}{\hookrightarrow} V_{1} u\left(F_{1}, F_{2}\right) \in V_{1,2}$, причем $F_{2} \in L_{V_{2}}\left(X_{2}\right)$. Тогда для любого компакта $Y \subset\left(X_{1} \backslash X_{2}\right)$ и любого $\varepsilon>0$ найдутся $h_{0} \in V_{1}$ и окрестность $U_{2}$ компакта $X_{2}$, удовлетворяющие следующим условиям:

1) $\operatorname{supp} h_{0}-$ компакт;

2) $h_{0}^{(1)}=F_{1(Y)}^{(1)}$

3) $L h_{0}=0$ в $U_{2}$;

4) $\left\|\left(h_{0}, h_{0}\right)-\left(F_{1}, F_{2}\right)\right\|_{1,2}<\varepsilon$.

ДоКАЗАТЕЛЬСТво. Фиксируем произвольное $\eta>0$ и из условия $F_{2} \in L_{V_{2}}\left(X_{2}\right)$ найдем функцию $g_{\eta} \in L\left(X_{2}\right) \cap C_{0}^{\infty}$ такую, что $\left\|F_{2}-g_{\eta}\right\|_{2, X_{2}}<\eta$. Также для этого $\eta>0$ возьмем $f_{0}$ и $\varphi$ из доказательства предложения 1 . Используя последнюю оценку и то, что $f_{0} \underset{\left(X_{2}\right)}{(2)}=F_{2}$, можно найти распределение $f_{\eta}$ и окрестность $U_{2}$ (зависящую от $\eta$ ) компакта $X_{2}$ такие, что $f_{\eta} \in V_{2}, f_{\eta}=f_{0}-g_{\eta}$ в $U_{2},\left\|f_{\eta}\right\|_{2} \leqslant\left\|f_{0}-g_{\eta}\right\|_{2, X_{2}}+\eta<2 \eta$ и функция $\varphi \equiv 1$ в $U_{2}$.

Положим $h_{0}=f_{0}-f_{\eta} \varphi$. Тогда $h_{0}-$ распределение с компактным носителем, $h_{0} \in V_{1}$, $h_{0} \in V_{2, \text { lос }}\left(U_{2}\right)$ и $h_{0}^{(1)}=f_{0}^{(1)}=F_{1(Y)}^{(1)}$. Далее, $L h_{0}=L f_{0}-L\left(\varphi f_{\eta}\right)=0$ в $U_{2}$. Воспользуемся вложением $V_{2} \stackrel{\text { loc }}{\hookrightarrow} V_{1}$ и свойством (2) для $V_{2}$. Имеем

$$
\left\|h_{0}-F_{1}\right\|_{1, X_{1}} \leqslant\left\|h_{0}-f_{0}\right\|_{1, X_{1}}+\left\|f_{0}-F_{1}\right\|_{1, X_{1}} \leqslant\left\|f_{\eta} \varphi\right\|_{1, X_{1}}+\eta \leqslant\left(A_{1}+1\right) \eta,
$$

где $A_{1}>0$ зависит лишь от $\varphi$ и $X_{1}$,

$$
\left\|h_{0}-F_{2}\right\|_{2, X_{2}}=\left\|f_{0}-F_{2}-f_{\eta} \varphi\right\|_{2, X_{2}} \leqslant\left\|f_{0}-F_{2}\right\|_{2, X_{2}}+\left\|f_{\eta} \varphi\right\|_{2, X_{2}} \leqslant A_{2} \eta,
$$

где $A_{2}>0$ зависит лишь от $\varphi$. Следовательно, для каждого $\varepsilon>0$, выбирая $\eta=$ $\varepsilon\left(\max \left(A_{1}+1, A_{2}\right)\right)^{-1}$, получаем нужное распределение $h_{0}$.

Рассуждения, аналогичные доказательству предложения 2 , приводят нас к следующему утверждению.

ПрЕДЛОЖЕНИЕ 3. Пусть $V_{2} \stackrel{\text { loc }}{\longrightarrow} V_{1} u\left(F_{1}, F_{2}\right) \in W_{1,2}$. Тогда для любого компакта $Y \subset\left(X_{1} \backslash X_{2}\right)$ и любого $\varepsilon>0$ найдется $f_{0} \in V_{1}$, удовлетворяющая следующим условиям: $\operatorname{supp} f_{0}-$ компакт, $L f_{0}=0$ в некоторой окрестности компакта $X_{2} \cup Y$ $u\left\|\left(f_{0}, f_{0}\right)-\left(F_{1}, F_{2}\right)\right\|_{1,2}<\varepsilon$.

Теперь мы сформулируем и докажем предложение, касающееся некоторых свойств локализационного оператора Витушкина. 
ПредлОЖЕнИЕ 4. Пусть $K-$ компакт в $\mathbb{R}^{N}, a \in \mathbb{R}^{N} \backslash K$. Тогда для любых $\delta \in(0, \operatorname{dist}(a, K)) u \varphi \in C_{0}^{\infty}(B(a, \delta))$ существует $A>0$ такая, ито

$$
\left\|\mathscr{V}_{\varphi} f\right\|_{2, K} \leqslant A\|f\|_{1, \bar{B}(a, \delta)} \quad \text { для всех } f \in V_{1, \mathrm{loc}}
$$

ДокАЗАТЕЛЬСтво. Фиксируем $\sigma \in(0, \operatorname{dist}(a, K))$ (которое выберем позднее), и пусть $K_{\sigma}=\left\{x \in \mathbb{R}^{N}: \operatorname{dist}(x, K) \leqslant \sigma\right\}-\sigma$-раздутие компакта $K$. Без ограничения обшности положим $a=0$. Пусть $\delta_{1}>0$ такое, что $\bar{B}\left(0, \delta_{1}\right)$ не пересекается с $K_{\sigma}$. Тогда для любых $\varphi \in C_{0}^{\infty}\left(B\left(0, \delta_{1}\right)\right)$ и $f \in V_{1, \text { loc из определения оператора Витушкина по- }}$ лучаем $L\left(\mathscr{V}_{\varphi} f\right)=0$ вне $\bar{B}\left(0, \delta_{1}\right)$, т.е. имеет смысл норма $\left\|\mathscr{V}_{\varphi} f\right\|_{2, K}$. Далее, выберем

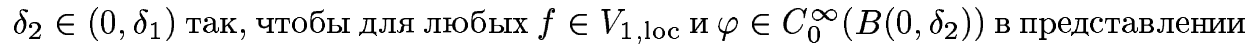

$$
\mathscr{V}_{\varphi} f(x)=\sum_{|\alpha| \geqslant 0} c_{\alpha} \partial^{\alpha} \Phi(x), \quad \text { где } x \in \mathbb{R}^{N} \backslash \bar{B}\left(0, \delta_{2}\right),
$$

ряд справа сходился в $C^{\infty}\left(|x|>\delta_{2}\right)$.

Для коэффициентов ряда (4) по правилу дифференцирования распределений имеем

$$
\left|c_{\alpha}\right|=\left|(\alpha !)^{-1}\left\langle\varphi(y) L f, y^{\alpha}\right\rangle\right|=(\alpha !)^{-1}\left|\left\langle f, L\left(\varphi(y) y^{\alpha}\right)\right\rangle\right| .
$$

Чтобы оценить коэффициенты $c_{\alpha}$, воспользуемся следующей теоремой из теории распределений (см., например, [15, с. 22]).

Теорема А. Пусть множество $M \subset\left(C_{0}^{\infty}\right)^{*}$ слабо ограничено (т.е. для любого $\varphi \in C_{0}^{\infty}$ uf $\in$ м имеем $|\langle f, \varphi\rangle| \leqslant A_{\varphi}$, где $A_{\varphi}$ не зависит от $\left.f\right)$. Тогда для каждого открытого шара $B$ в $\mathbb{R}^{N}$ найдутся числа $A_{1}=A_{1}(B, M)>0$ и $m=m(B, M) \in \mathbb{Z}_{+}$ такие, что оченка

$$
|\langle f, \varphi\rangle| \leqslant A_{1}\|\varphi\|_{C^{m}(\bar{B})}
$$

верна для всех $\varphi \in C_{0}^{\infty}(B)$ и $f \in M$, где $\|\cdot\|_{C^{m}(\bar{B})}-$ стандартная норма в $C^{m}(\bar{B})$ (см. пример 2 из n. 4.1).

Применим это утверждение к открытому шару $B=B(0, \delta)$ для некоторого $\delta \in\left(0, \delta_{2}\right)$ (которое выберем позже) и множеству $M=\left\{h \in V_{1}:\|h\|_{1}=1\right\}$, слабая ограниченность которого следует из свойства (2) для $V_{1}$. Тогда найдутся числа $A_{1}=A_{1}(B, M)>0$ и $m=m(B, M) \in \mathbb{Z}_{+}$такие, что

$$
\left|\left\langle h, L\left(\varphi(y) y^{\alpha}\right)\right\rangle\right| \leqslant A_{1}\left\|L\left(\varphi(y) y^{\alpha}\right)\right\|_{C^{m}(\bar{B})}
$$

для всех $h \in M$ и $\varphi \in C_{0}^{\infty}(B)$.

Используя формулу Лейбница, несложно получить, что

$$
\left\|L\left(\varphi(y) y^{\alpha}\right)\right\|_{C^{m}(\bar{B})} \leqslant A_{2}|\alpha|^{m+r} \delta^{|\alpha|},
$$

где $A_{2}>0$ не зависит от $\alpha$ и $\delta$. Тогда для произвольного $\varphi \in C_{0}^{\infty}(B)$ имеем

$$
\left|\left\langle h, L\left(\varphi(y) y^{\alpha}\right)\right\rangle\right| \leqslant A_{3}\|h\|_{1}|\alpha|^{m+r} \delta^{|\alpha|}
$$


для любых $h \in V_{1}$, причем $A_{3}>0$ не зависит от $h, \delta$ и $\alpha$. При $p \in \mathbb{Z}_{+}$оценим ряд (4) в норме пространства $C^{p}\left(K_{\sigma}\right)$. В силу последней оценки

$$
\begin{aligned}
\left\|\mathscr{V}_{\varphi} h\right\|_{C^{p}\left(K_{\sigma}\right)} & \leqslant \sum_{|\alpha| \geqslant 0}\left|c_{\alpha}\right|\left\|\partial^{\alpha} \Phi(x)\right\|_{C^{p}\left(K_{\sigma}\right)} \\
& \leqslant A_{3}\|h\|_{1} \sum_{|\alpha| \geqslant 0}(\alpha !)^{-1}|\alpha|^{m+r} \delta^{|\alpha|}\left\|\partial^{\alpha} \Phi(x)\right\|_{C^{p}\left(K_{\sigma}\right)}
\end{aligned}
$$

Taк как (см. [4, с. 254])

$$
\left|\partial^{\alpha} \Phi(x)\right| \leqslant \alpha ! A_{4}^{|\alpha|}|x|^{-(N-r+|\alpha|)} \cdot(|\log | x||+1), \quad x \neq 0,
$$

где $A_{4}>1-$ константа, зависящая лишш от вида оператора $L$, то при $d=\operatorname{dist}\left(0, K_{\sigma}\right)$ имеем

$$
\left\|\mathscr{V}_{\varphi} h\right\|_{C}\left(K_{\sigma}\right) \leqslant A_{5}\|h\|_{1} \sum_{|\alpha| \geqslant 0}|\alpha|^{m+r}(\alpha !)^{-1}\left(\alpha_{1}+p\right) ! \cdots\left(\alpha_{N}+p\right) !\left(\frac{A_{4} \delta}{d}\right)^{|\alpha|}
$$

где $A_{5}>0$ не зависит от $h$. При $\delta \in\left(0, d / A_{4}\right)$ ряд справа будет сходиться; следовательно, для $\varphi \in C_{0}^{\infty}(B(0, \delta))$ имеет место оценка

$$
\left\|\mathscr{V}_{\varphi} h\right\|_{C^{p}\left(K_{\sigma}\right)} \leqslant A_{6}\|h\|_{1}
$$

и $A_{6}>0$ не зависит от $h$.

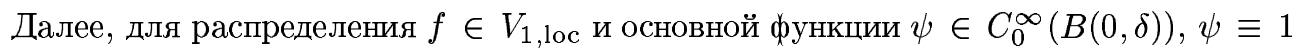
на $\operatorname{supp} \varphi$, имеем

$$
\mathscr{V}_{\varphi} f=\Phi * \varphi L f=\Phi * \varphi L(\psi f)=\mathscr{V}_{\varphi}(\psi f)
$$

Так как $\psi f \in V_{1}$, то

$$
\left\|\mathscr{V}_{\varphi}(\psi f)\right\|_{C^{p}\left(K_{\sigma}\right)} \leqslant A_{6}\|\psi f\|_{1}
$$

откуда

$$
\left\|\mathscr{V}_{\varphi} f\right\|_{C^{p}\left(K_{\sigma}\right)} \leqslant A_{7}\|f\|_{1, \bar{B}(0, \delta)}
$$

и $A_{7}>0$ не зависит от $f$, но может зависеть от $p$.

Покажем, что последняя оценка верна с заменой $\|\cdot\|_{C^{p}\left(K_{\sigma}\right)}$ на $\|\cdot\|_{2, K}$.

Заметим, что поскольку вложение $C^{\infty} \hookrightarrow V_{2}$, ос непрерьвно, для $K$ найдется $\sigma \in$ $(0, \operatorname{dist}(0, K))$ такое, что отображение $\omega \rightarrow \omega_{(K)}$ непрерывно из $C^{\infty}\left(K_{\sigma}\right)$ в $V_{2, \text { loc }}(K)$. Пространство $C^{\infty}\left(K_{\sigma}\right)$ является счетно-нормированным пространством, топология в котором может быть задана при помощи следующей метрики (инвариантной относительно сдвигов):

$$
\rho(f, g)=\sum_{p=0}^{\infty} 2^{-p} \frac{\|f-g\|_{p}}{1+\|f-g\|_{p}}
$$

где $f, g \in C^{\infty}\left(K_{\sigma}\right)$ и $\|\cdot\|_{p}=\|\cdot\|_{C^{p}\left(K_{\sigma}\right)}$. Положим $\lambda_{p}=\max \left(A_{7}(p), 1\right)$ для всех $p \in \mathbb{Z}_{+}$. Метрика

$$
\widetilde{\rho}(f, g)=\sum_{p=0}^{\infty} \frac{2^{-p}}{\lambda_{p}} \frac{\|f-g\|_{p}}{1+\|f-g\|_{p}}
$$


эквивалентна метрике $\rho$; следовательно, непрерьвность линейного отображения $\omega \rightarrow$ $\omega_{(K)}$ означает, что существует $A_{8}>0$ такая, что $\|\omega\|_{2, K} \leqslant A_{8} \widetilde{\rho}(0, \omega)$, причем $A_{8}$ не зависит от $\omega$. Тогда

$$
\begin{aligned}
\left\|\mathscr{V}_{\varphi} f\right\|_{2, K} & \leqslant A_{8} \sum_{p=0}^{\infty} \frac{2^{-p}}{\lambda_{p}} \frac{\left\|\mathscr{V}_{\varphi} f\right\|_{C}\left(K_{\sigma}\right)}{1+\left\|\mathscr{V}_{\varphi} f\right\|_{C}\left(K_{\sigma}\right)} \\
& \leqslant A_{8} \sum_{p=0}^{\infty} \frac{A_{7}(p)}{\lambda_{p}} 2^{-p}\|f\|_{1, \bar{B}\left(0, \delta_{0}\right)} \leqslant A_{9}\|f\|_{1, \bar{B}\left(0, \delta_{0}\right)}
\end{aligned}
$$

и $A_{9}>0$ не зависит от $f$.

Итак, мы показали, что для каждой $a \notin K$ найдутся $\delta_{0} \in(0, \operatorname{dist}(a, K))$ и $A_{9}>0$ такие, что для любой $\varphi \in C_{0}^{\infty}\left(B\left(a, \delta_{0}\right)\right)$ выполняется

$$
\left\|\mathscr{V}_{\varphi} f\right\|_{2, K} \leqslant A_{9}\|f\|_{1, \bar{B}\left(a, \delta_{0}\right)}
$$

для всех $f \in V_{1, \mathrm{loc}}$, причем $A_{9}$ не зависит от $f$.

Пусть теперь $B=B(a, \delta)$ - произвольньй шар с условием $\delta \in(0, \operatorname{dist}(a, K))$ и $\varphi \in$ $C_{0}^{\infty}(B)$. Тогда для каждой $x \in \bar{B}$ найдется $\delta_{x} \in(0, \operatorname{dist}(x, K))$ и $A_{x}>0$ такие, что для любого $\psi_{x} \in C_{0}^{\infty}\left(B_{x}\right)$ (где $B_{x}=B\left(x, \delta_{x}\right)$ ) имеем

$$
\left\|\mathscr{V}_{\psi_{x}} f\right\|_{2, K} \leqslant A_{x}\|f\|_{1, \bar{B}_{x}}
$$

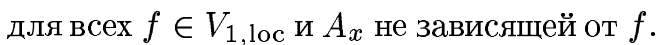

Система $\left\{B_{x}\right\}_{x \in \bar{B}}$ - открытое покрытие компакта $\bar{B}$; следовательно, из него может быть выделено конечное подпокрытие $\left\{B_{x_{j}}\right\}_{j=1}^{J}$. Положим $B_{j}:=B_{x_{j}}$. Для покрытия $\left\{B_{j}\right\}_{j=1}^{J}$ рассмотрим систему функций $\left\{\psi_{j}\right\}_{j=1}^{J}$ таких, что

$$
\psi_{j} \in C_{0}^{\infty}\left(B_{j}\right), \quad 0 \leqslant \psi_{j} \leqslant 1, \quad j \in\{1,2, \ldots, J\}, \quad \sum_{j=1}^{J} \psi_{j}(x) \equiv 1
$$

в некоторой окрестности $\bar{B}$. Тогда имеет место равенство

$$
\mathscr{V}_{\varphi} f=\sum_{j=1}^{J} \mathscr{V}_{\varphi \psi_{j}} f
$$

Следовательно,

$$
\begin{aligned}
\left\|\mathscr{V}_{\varphi} f\right\|_{2, K} & =\left\|\sum_{j=1}^{J} \mathscr{V}_{\varphi \psi_{j}} f\right\|_{2, K} \leqslant \sum_{j=1}^{J}\left\|\mathscr{V}_{\varphi \psi_{j}} f\right\|_{2, K} \\
& \leqslant \sum_{j=1}^{J} A_{x, j}\|f\|_{1, \bar{B}_{j} \cap \operatorname{supp} \varphi} \leqslant \sum_{j=1}^{J} A_{x, j}\|f\|_{1, \bar{B}} .
\end{aligned}
$$

Последняя оценка завершает доказательство предложения 4. 
ЗАмЕчАниЕ 3. Отметим, что в предложении 4 условие локального вложения $V_{2} \stackrel{\text { loc }}{\hookrightarrow} V_{1}$ не требуется.

В доказательстве теоремы 3 нам потребуется следующее уточнение условия (ПК).

ПРЕДЛОЖЕНИЕ 5. Пусть $\left(V_{1}, L\right)$ удовлетворяет условию (3), а $X_{1} u X_{2}$ удовлетворяют условию (ПК). Тогда для любих $\varepsilon>0$, иара $B(a, \delta)$ такого, что

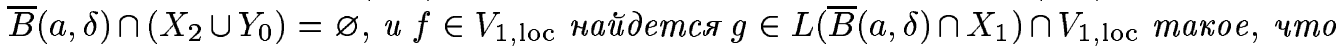
$\|f-g\|_{1}<\varepsilon$.

ДокАЗАТЕЛЬСтво. Считаем, что $\bar{B}(a, \delta) \cap X_{1} \neq \varnothing$. По условию (ПК) (см. замечание 1) для любых $\eta>0$ и $x \in \bar{B}(a, \delta) \cap X_{1}$ найдутся $p_{x} \geqslant 1, \delta_{x} \in\left(0, \operatorname{dist}\left(x, X_{2} \cup Y_{0}\right) / p_{x}\right)$ и $g_{\eta, x} \in L\left(\bar{B}\left(x, \delta_{x}\right) \cap X_{1}\right) \cap C_{0}^{\infty}$ такие, что

$$
\left\|f-g_{\eta, x}\right\|_{1, \bar{B}}\left(x, \delta_{x}\right) \cap X_{1}<\frac{\eta}{2} .
$$

Из открытого покрытия $\left\{B\left(x, \delta_{x}\right)\right\}_{x \in \bar{B}}(a, \delta) \cap X_{1}$ компакта $\bar{B}(a, \delta) \cap X_{1}$ выделим конечное подпокрытие $\left\{B\left(x_{j}, \delta_{j}\right)\right\}_{j=1}^{J}$ и найдем систему функций $\left\{\varphi_{j}\right\}_{j=1}^{J}$, удовлетворяющих условиям

$$
\varphi_{j} \in C_{0}^{\infty}\left(B\left(x_{j}, \delta_{j}\right)\right), \quad 0 \leqslant \varphi_{j} \leqslant 1, \quad j \in\{1,2, \ldots, J\}, \quad \sum_{j=1}^{J} \varphi_{j}(x)=1
$$

в некоторой окрестности $\bar{B}(a, \delta) \cap X_{1}$.

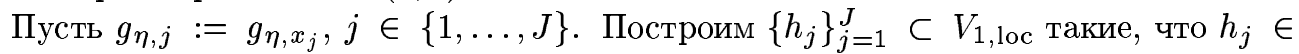
$L\left(\bar{B}\left(x_{j}, \delta_{j}\right) \cap X_{1}\right)$ и $\left\|f-h_{j}\right\|_{1}<\eta$. По определению нормы в $V_{1}\left(\bar{B}\left(x_{j}, \delta_{j}\right) \cap X_{1}\right)$ мы можем найти окрестность $U_{j}$ компакта $\bar{B}\left(x_{j}, \delta_{j}\right) \cap X_{1}$, в которой $L g_{\eta, j}=0$ и $\left\|f-g_{\eta, j}\right\|_{1, \bar{U}_{j}}<\eta$. Пусть $\widetilde{h}_{j} \in V_{1}$ такой, что $f-g_{\eta, j}=\widetilde{h}_{j}$ в $U_{j}$ и $\left\|\widetilde{h}_{j}\right\|_{1}<\eta$. Тогда распределения $h_{j}=f-\widetilde{h}_{j}, j \in\{1, \ldots, J\}$, искомые.

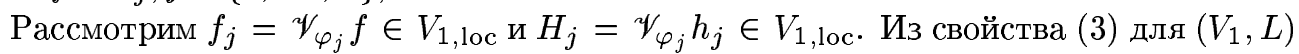
получаем

$$
\left\|f_{j}-H_{j}\right\|_{1, X}=\left\|\mathscr{V}_{\varphi_{j}}\left(f-h_{j}\right)\right\|_{1, X} \leqslant A_{j}\left\|f-h_{j}\right\|_{1}<A_{j} \eta
$$

где $A_{j}=A\left(\varphi_{j}, B\right)$ из (3). Положим

$$
g=f-\sum_{j=1}^{J}\left(f_{j}-H_{j}\right)
$$

Тогда

$$
L g=L\left(f-\sum_{j=1}^{J} f_{j}\right)+\sum_{j=1}^{J} L H_{j}=L f\left(1-\sum_{j=1}^{J} \varphi_{j}\right)+\sum_{j=1}^{J} \varphi_{j} L h_{j}=0
$$

в некоторой окрестности $\bar{B}(a, \delta) \cap X_{1}$. Откуда $g \in L\left(\bar{B}(a, \delta) \cap X_{1}\right) \cap V_{1, \text { loc и }}$

$$
\|f-g\|_{1, X}<\eta \sum_{j=1}^{J} A_{j} .
$$

Полагая $\eta=\varepsilon /\left(\sum_{j=1}^{J} A_{j}\right)$, получаем $\|f-g\|_{1, X}<\varepsilon$. Для завершения доказательства остается заметить, что рассуждения, приведенные чуть вьше для построения $h_{j}$, пока-

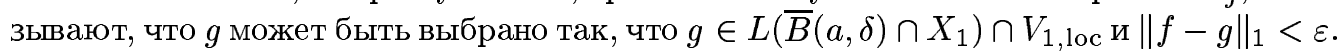


3.4. Доказательство теоремы 3. Пусть $\left(F_{1}, F_{2}\right) \in W_{1,2}$ и $\eta>0$ произвольно. Из условия (ПК) и предложения 3 следует, что найдется $f_{0} \in V_{1}$ с компактным носителем

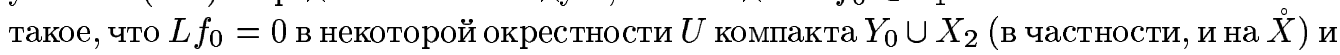
$\left\|\left(f_{0}, f_{0}\right)-\left(F_{1}, F_{2}\right)\right\|_{1,2}<\eta$. Для доказательства теоремы 3 остается для произвольного $\varepsilon>0$ найти $G \in L(X)$, удовлетворяющее условию $\left\|\left(f_{0}, f_{0}\right)-(G, G)\right\|_{1,2}<\varepsilon$. Фиксируем $\eta, f_{0}, U, \varepsilon$ и опишем построение $G$. Пусть $j=\left(j_{1}, \ldots, j_{N}\right) \in \mathbb{Z}^{N}$. Для

$$
\delta \in\left(0, \frac{1}{10 A_{4}} \cdot \operatorname{dist}\left(\partial U, X_{2} \cup Y_{0}\right)\right),
$$

где $A_{4}$ взято из (5), рассмотрим $\delta$-решетку $\left\{a_{j}=\left(j_{1} \delta, \ldots, j_{N} \delta\right)\right\}_{j \in \mathbb{Z}^{N}}$ и систему открытых шаров $\left\{B_{j}=B\left(a_{j}, \delta \sqrt{N}\right)\right\}_{j \in \mathbb{Z}^{N}}$, покрывающую $\mathbb{R}^{N}$. Далее, пусть $\left\{\varphi_{j}\right\}_{j \in \mathbb{Z}^{N}}$ $\left(\varphi_{j} \in C_{0}^{\infty}\left(B_{j}\right), 0 \leqslant \varphi_{j} \leqslant 1, \sum_{j \in \mathbb{Z}^{N}} \varphi_{j}(x) \equiv 1\right)$ - разбиение единицы, ассоциированное с покрытием $\left\{B_{j}\right\}_{j \in \mathbb{Z}^{N}}$. Положим $f_{j}=\mathscr{V}_{\varphi_{j}} f_{0} \in V_{1, \text { loc }}$. Тогда если $B_{j} \subset U$ или $\bar{B}_{j} \cap \operatorname{supp} f_{0}=\varnothing$, то $f_{j} \equiv 0$. Также если $B_{j} \subset \mathbb{R}^{N} \backslash X$, то $L f_{j} \equiv 0$ в окрестности $X$.

Предположим, что $X \backslash U \neq \varnothing$ (иначе $f_{0}$ - требуемое приближение) и обозначим $J=\left\{j \in \mathbb{Z}^{N}: \bar{B}_{j} \cap \overline{(X \backslash U)} \neq \varnothing\right\}$. Множество $J$ конечно, причем в силу выбора $\delta$ для всех $j \in J$ имеем $\bar{B}_{j} \cap\left(X_{2} \cup Y_{0}\right)=\varnothing$. При каждом $j \in J$ и $\varepsilon_{0}$ (которое выберем позже)

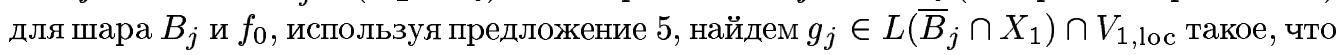
$\left\|f_{0}-g_{j}\right\|_{1}<\varepsilon_{0}$. Положим $G_{j}=\mathscr{V}_{\varphi_{j}} g_{j} \in V_{1, \text { loc }}$ и рассмотрим

$$
G=f_{0}-\sum_{j \in J}\left(f_{j}-G_{j}\right) \in V_{1, \mathrm{loc}}
$$

Тогда $G \in L(X)$ (см. доказательство предложения 5), причем из (3) получаем

$$
\left\|G-f_{0}\right\|_{1, X_{1}} \leqslant \sum_{j \in J}\left\|f_{j}-G_{j}\right\|_{1, X_{1}} \leqslant \sum_{j \in J} A_{j}^{(1)}\left\|f_{0}-g_{j}\right\|_{1}<\varepsilon_{0} \sum_{j \in J} A_{j}^{(1)},
$$

где $A_{j}^{(1)}$ зависят только от $\varphi_{j}$ и $B_{j}$. Далее,

$$
\left\|G-f_{0}\right\|_{2, X_{2}} \leqslant \sum_{j \in J}\left\|f_{j}-G_{j}\right\|_{2, X_{2}}=\sum_{j \in J}\left\|\mathscr{V}_{\varphi_{j}}\left(f_{0}-g_{j}\right)\right\|_{2, X_{2}} .
$$

В силу выбора $\delta$ из предложения 4 находим

$$
\left\|\mathscr{V}_{\varphi_{j}}\left(f_{0}-g_{j}\right)\right\|_{2, X_{2}} \leqslant A_{j}^{(2)}\left\|f_{0}-g_{j}\right\|_{1, \bar{B}_{j}},
$$

где $A_{j}^{(2)}$ не зависят от $\varepsilon_{0}$, откуда

$$
\left\|G-f_{0}\right\|_{2, X_{2}}<\varepsilon_{0} \sum_{j \in J} A_{j}^{(2)} .
$$

Полагая

$$
\varepsilon_{0}=\frac{\varepsilon}{\max \left(\sum_{j \in J} A_{j}^{(1)}, \sum_{j \in J} A_{j}^{(2)}\right)},
$$

получаем требуемое распределение $G$. 


\section{4. Некоторые примеры и следствия}

4.1. Примеры применения теорем 3 и 4. Здесь мы сформулируем следствия доказанных нами теорем только для аппроксимации некоторых классов функций, поскольку формулировки этих следствий для индивидуальных функций рассматриваемьх классов немедленно вытекают из теорем 3 и 4 и соответствующих критериев аппроксимации в каждом $V_{s}$.

Пример 1. Пусть $E$ - замкнутое подмножество в $\mathbb{R}^{N}, t \in(0,1)$. Банахово пространство $\operatorname{lip}_{t}(E)$ определяется как класс всех функций $f: E \rightarrow \mathbb{C}$, удовлетворяющих следующим условиям:

$$
\|f\|_{0, E}=\sup _{x \in E}|f(x)|<\infty, \quad\|f\|_{t, E}^{\prime}=\sup _{\delta>0} \frac{\omega_{E}(f, \delta)}{\delta^{t}}<\infty,
$$

$\omega_{E}(f, \delta)=o\left(\delta^{t}\right)$ при $\delta \rightarrow 0$, где $\omega_{E}(f, \cdot)$ - модуль непрерьвности $f$ на $E$.

Норма в этом пространстве задается равенством

$$
\|f\|_{t, E}=\|f\|_{0, E}+\|f\|_{t, E}^{\prime} .
$$

При $E=\mathbb{R}^{N}$ индексы $E$ в записи норм опускаем и полагаем $\operatorname{lip}_{t}=\operatorname{lip}_{t}\left(\mathbb{R}^{N}\right)$. Условия (1) и (2) для пространств $\operatorname{lip}_{t}, t \in(0,1)$, проверяются элементарно. Следовательно, $\operatorname{lip}_{t}-$ КБП. По теореме Уитни $\left[16\right.$, с. 206] для произвольного компакта $K$ в $\mathbb{R}^{N}$ пространство струй $\operatorname{lip}_{t}(K)$ изоморфно введенному вьше пространству функций $\operatorname{lip}_{t}(K)$.

Фиксируем произвольные $\alpha$ и $\beta$, удовлетворяющие $0<\alpha<\beta<1$, и рассмотрим задачу о совместных приближениях в пространствах $V_{1}=\operatorname{lip}_{\alpha}$ и $V_{2}=\operatorname{lip}_{\beta}$ в $\mathbb{R}^{2}$, причем в качестве эллиптического оператора $L$ возьмем оператор Коши-Римана $\partial / \partial \bar{z}$ в $\mathbb{C}_{z}$. Нетрудно непосредственно установить, что $V_{2} \stackrel{\text { loc }}{\hookrightarrow} V_{1}$ и для пары $\left(V_{1}, L\right)$ (а также и для пары $\left.\left(V_{2}, L\right)\right)$ выполнено (3) (см. [9]).

Пусть $X_{1}$ и $X_{2}$ - компакты в $\mathbb{R}^{2}$ (напомним, что $X=X_{1} \cup X_{2}, X_{0}=X_{1} \cap X_{2} \neq \varnothing$ ). Тогда

$$
\begin{aligned}
V_{1,2} & =\left\{\left(f_{1}, f_{2}\right) \mid f_{1} \in \operatorname{lip}_{\alpha}\left(X_{1}\right), f_{2} \in \operatorname{lip}_{\beta}\left(X_{2}\right) \text { и }\left.f_{1}\right|_{X_{0}}=\left.f_{2}\right|_{X_{0}}\right\}, \\
H_{1,2} & =\left\{\left(f_{1}, f_{2}\right) \in V_{1,2} \mid f_{s} \text { голоморфнана н } \stackrel{\circ}{X}_{s}, s \in\{1,2\}\right\} .
\end{aligned}
$$

ДоКАЗАТЕЛЬСТво ТЕОРЕмЫ 1. Достаточно воспользоваться теоремой 4 (см. п. 3.2) и теоремой О'Фаррелла [17]. Заметим, что в теореме 1 условие (ПК) не требуется, поскольку оно автоматически следует из (ii).

ПримеР 2. В этом примере положим $V_{1}=B C^{0}\left(\mathbb{R}^{2}\right), V_{2}=B C^{1}\left(\mathbb{R}^{2}\right)$ и $L=\partial / \partial \bar{z}$, где $B C^{m}\left(\mathbb{R}^{N}\right), m \in \mathbb{Z}_{+}$, - пространство всех $m$ раз непрерьвно дифференцируемых функций $f: \mathbb{R}^{N} \rightarrow \mathbb{C}$ с конечной нормой

$$
\|f\|_{m}=\sum_{|\alpha| \leqslant m} \sup _{x \in \mathbb{R}^{N}}\left|\partial^{\alpha} f(x)\right| .
$$

Вьполнение условий $(1),(2)$ для $V_{1}$ и $V_{2}$ проверяется элементарно. Справедливость (3) для $\left(V_{1}, L\right)$ следует из $[8$, гл. $2, \S 3]$. Также очевидно, что $V_{2} \stackrel{\text { loc }}{\hookrightarrow} V_{1}$. 
Для произвольного компакта $K$ в $\mathbb{R}^{2}$ пространство $B C^{0}(K)$ (которое будем обозначать $C(K))$ есть пространство непрерывных функций на $K$ с равномерной нормой $\|\cdot\|_{0, K}$. Пространство $B C^{1}(K)$ (его будем обозначать $C^{1}(K)$ ) определяется выше в разделе 2 как факторпространство $B C^{1}\left(\mathbb{R}^{2}\right) / I(K)$, где $I(K)$ есть замыкание в $B C^{1}\left(\mathbb{R}^{2}\right)$ линейного подмногообразия $\left\{f \in B C^{1}\left(\mathbb{R}^{2}\right): \operatorname{supp} f \cap K=\varnothing\right\}$. Последнее определение по теореме Уитни [16, с. 210] эквивалентно более наглядному определению пространства $C^{1}(K)$, данному в разделе 1.

Пусть, как и ранее, $X_{1}$ и $X_{2}$ - непустые компакты в $\mathbb{R}^{2}$. Тогда

$$
\begin{aligned}
& V_{1,2}=\left\{\left(f_{1}, F_{2}\right) \mid f_{1} \in C\left(X_{1}\right), F_{2}=\left(h_{0}, h_{1}, h_{2}\right) \in C^{1}\left(X_{2}\right) \text { и } f_{1}\left|x_{0}=h_{0}\right| x_{0}\right\}, \\
& H_{1,2}=\left\{\left(f_{1}, F_{2}\right) \in V_{1,2} \mid f_{1} \text { голоморфна на } \stackrel{\circ}{X}_{1}, h_{0} \text { голоморфна на } \stackrel{\circ}{2}_{2}\right\} .
\end{aligned}
$$

ДокАЗАТЕЛЬСтво тЕоремЫ 2. Следует воспользоваться теоремой Витушкина [8, гл. $2, \S 3]$, результатом Дж. Вердеры [7, теорема 1] и теоремой 4 (см. также [5, теорема 4 (ii)]). Так же, как и в примере 1 , здесь условие (ПК) следует из (ii).

Другие примеры наличия и отсутствия совместных приближений, подтверждающие “существенность” условий теорем 3 и 4, планируется привести в последующей работе.

4.2. Совместные приближения на нигде не плотных компактах в $\mathbb{R}^{N}$. Фиксируем КБП $V$ и оператор $L$.

УСловие (А). Скажем, что для непустого компакта $K$ в $\mathbb{R}^{N}$ и пары $(V, L)$ вьполнено yсловие (A), если найдется полином $p$ такой, что $L p \equiv 1$ и $p_{(K)} \in L_{V}(K)$.

Очевидно, что условию (А) могут удовлетворять только нигде не плотные компакты $K$ в $\mathbb{R}^{N}$ и одновременно все полиномы с условием $L p \equiv 1$. Для таких компактов верна следующая теорема, частные случаи которой можно найти в [10]-[12].

ПРЕДЛОЖЕНИЕ 6. Пусть $K$ - компакт в $\mathbb{R}^{N}$, удовлетворяющий условию (A). Пусть $C^{\infty}$ плотно в $V_{\mathrm{loc}} u(V, L)$ удовлетворяет $(3)$. Тогда $V(K)=L_{V}(K)$.

ДокАЗАтЕльство. Пусть $F \in V(K)$ и $f \in F$ - некоторьй представитель класса $F$, причем такой, что supp $f$ - компакт. Предположим, что $\operatorname{supp} f \cup K \subset B=B(0, R)$, где $R>0$. Фиксируем $\varepsilon>0$. По условию найдется $\varphi \in C_{0}^{\infty}(B)$ такая, что $\|\varphi-f\|_{K}<\varepsilon / 2$.

Пусть $p$ - полином такой, что $L p \equiv 1$, и для любого $\eta>0$ найдется $g_{\eta} \in L(K) \cap V_{\text {loc }}$ такой, что $\left\|p-g_{\eta}\right\|_{K}<\eta$. По определению нормы $\|\cdot\|_{K}$ можно добиться неравенства $\left\|p-g_{\eta}\right\|_{\bar{B}}<\eta$ (см. доказательство предложения 5). Пусть далее $\psi \in C_{0}^{\infty}, \psi \equiv 1$ на шаре $B(0, R)$. Тогда из равенства (см. [4, с. 255])

$$
\varphi=\Phi * L \varphi=\Phi *[L \varphi L(\psi p)]=\mathscr{V}_{L \varphi}(\psi p)
$$

и свойства (3) находим

$$
\begin{aligned}
\left\|\varphi-\mathscr{V}_{L \varphi}\left(\psi g_{\eta}\right)\right\|_{K} & =\left\|\mathscr{V}_{L \varphi}\left(\psi p-\psi g_{\eta}\right)\right\|_{K} \leqslant\left\|\mathscr{V}_{L \varphi}\left(\psi p-\psi g_{\eta}\right)\right\|_{\bar{B}} \\
& \leqslant A_{1}\left\|\psi p-\psi g_{\eta}\right\|_{\bar{B}} \leqslant A_{2}\left\|p-g_{\eta}\right\|_{\bar{B}} \leqslant A_{2} \eta,
\end{aligned}
$$

где $A_{1}=A_{1}(\varphi, B)>0$ и $A_{2}=A_{2}(\varphi, \psi, B)>0$ не зависят от $\eta$. Положим $\eta=\varepsilon /\left(2 A_{2}\right)$. Поскольку $h=\mathscr{V}_{L \varphi}\left(\psi g_{\eta}\right) \in L(K) \cap V_{\text {lос и }}$

$$
\|F-h\|_{K} \leqslant\|f-\varphi\|_{K}+\|\varphi-h\|_{K}<\frac{\varepsilon}{2}+\frac{\varepsilon}{2}=\varepsilon,
$$

в силу произвольности $\varepsilon f \in L_{V}(K)$.

В качестве следствия теоремы 3 и предложения 6 получаем 
СлЕДСТВИЕ 1. Пусть для каждого $s \in\{1,2\} \quad V_{s}-$ КБП, пара $\left(V_{s}, L\right)$ удовле-

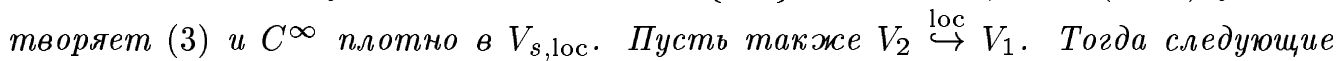
условия әквивалентны:

(i) при каждом $s \in\{1,2\}$ для компакта $X_{s}$ u пары $\left(V_{s}, L\right)$ выполнено условие $(\mathrm{A})$;

(ii) $V_{1,2}\left(X_{1}, X_{2}\right)=L_{1,2}\left(X_{1}, X_{2}\right)$.

Автор искренне признателен П. В. Парамонову и К. Ю. Федоровскому за внимание к работе.

\section{СПИСОК ЦИТИРОВАННОЙ ЛИТЕРАТУРЫ}

[1] Rubel L.A., Stray A. Joint approximation in the unit disc // J. Approximation Theory. 1983. V. 37. № 1. P. 44-50.

[2] Gonzalez F. P., Stray A. Farrell and Mergelyan sets for $H^{p}$ spaces // Michigan Math. J. 1989. V. 36. P. 379-386.

[3] Даниелян А. А. О некоторых задачах, возникающих из проблемы Рубеля об одновременной аппроксимации // Докл. РАН. 1995. Т. 341. №1. С. 10-12.

[4] Paramonov P. V., Verdera J. Approximation by solutions of elliptic equations on closed subsets of Euclidean space // Math. Scand. 1994. V. 74. P. 249-259.

[5] Бовен А., Парамонов П. В. Аппроксимация мероморфными и целыми решениями эллиптических уравнений в банаховых пространствах распределений // Матем. сб. 1998. Т. 189. № 4. C. 481-502.

[6] Davie A. M., Øksendal B. K. Rational approximation on the union of sets // Proc. Amer. Math. Soc. 1971. V. 29. № 3. P. 581-584.

[7] Verdera J. $C^{m}$-approximation by solutions of elliptic equations and Calderon-Zygmund operators // Duke Math. J. 1987. V. 55. P. 157-187.

[8] Витушкин А. Г. Аналитическая емкость множеств в задачах теории приближений // УМН. 1967. T. 22. №6. C. 141-199.

[9] O’Farrell A. G. T-invariance // Proc. Roy. Irish Acad. 1992. V. 92 A. № 2. P. 185-203.

[10] Khavinson D. On uniform approximation by harmonic functions // Michigan Math. J. 1987. V. 34. P. 465-473.

[11] Готье П. М., Парамонов П. В. Аппроксимация гармоническими функциями в $C^{1}$-норме и гармонический $C^{1}$-поперечник компактных множеств в $\mathbb{R}^{n} / /$ Матем. заметки. 1994. Т. 53. № 4. C. 21-30.

[12] Горохов Ю. А. Аппроксимация гармоническими функциями в $C^{m}$-норме и гармоническая $C^{m}$-вместимость компактных множеств в $\mathbb{R}^{n} / /$ Матем. заметки. 1997. Т. 62 . № 3. С. 372-382.

[13] Хёрмандер Л. Анализ линейных дифференциальных операторов с частными призводными (в 4-х томах). Т. 1. М.: Мир, 1986.

[14] Хёрмандер Л. Анализ линейных дифференциальных операторов с частными призводными (в 4-х томах). Т. 2. М.: Мир, 1986.

[15] Владимиров В. С. Обобщенные функции в математической физике. М.: Наука, 1976.

[16] Стейн И.М. Сингулярные интегралы и дифференциальные свойства функций. М.: Мир, 1973.

[17] O'Farrell A. G. Hausdorff content and rational approximation in fractional Lipschitz norms // Trans. Amer. Math. Soc. 1977. V. 228. P. 187-206. 